\title{
L'organisation du travail en exploitations familiales d'élevage*
}

\section{Enseignements d'une analyse comparée conduite en France, en Amérique latine et au Vietnam}

\author{
Sylvie Cournut ${ }^{1}$ \\ Gérard Servière ${ }^{2}$ \\ Nathalie Hostiou ${ }^{3}$ \\ Sophie Chauvat ${ }^{4}$ \\ Benoît Dedieu ${ }^{3}$ \\ ${ }^{1}$ VetAgro Sup \\ Campus agronomique de Clermont \\ Unité mixte de recherche (UMR) Métafort \\ BP 35 \\ 63370 Lempdes \\ France \\ <s.cournut@vetagro-sup.fr> \\ ${ }^{2}$ Institut de l'élevage \\ 9, allée Pierre de Fermat \\ 63170 Aubière \\ France \\ <gerard.serviere@inst-elevage.asso.fr> \\ ${ }^{3}$ Inra Unité mixte de recherche (UMR) \\ Métafort \\ 63122 Saint Genès \\ Champanelle \\ France \\ $<$ nhostiou@clermont.inra.fr > \\ <benoit.dedieu@clermont.inra.fr> \\ ${ }^{4}$ Institut de l'élevage Supagro \\ 2, place Pierre Viala \\ 34060 Montpellier cedex 1 \\ <Sophie.Chauvat@inst-elevage.asso.fr>
}

Tirés à part : S. Cournut

\begin{abstract}
Résumé
S'intéresser au devenir des exploitations familiales d'élevage nécessite l'étude de leur organisation du travail, considérée comme une contextualisation particulière des interactions entre la composition de la famille, la combinaison et le dimensionnement des activités économiques (agricoles et non agricoles) et le fonctionnement technique de l'élevage. Cette étude s'appuie sur une analyse comparative de 19 fermes réparties dans quatre régions du monde (Pampa d'Argentine et d'Uruguay; Amazonie brésilienne; Ségala français; montagnes du Nord du Vietnam), et choisies avec nos partenaires locaux de recherche et développement. Elle décrit la diversité des formes d'organisation du travail et questionne le devenir de l'activité d'élevage. Le cadre d'analyse du travail combine des variables relatives aux caractéristiques structurelles et productives des élevages et d'autres décrivant la configuration du collectif de travail, l'aménagement des bâtiments et équipements, la conduite de l'élevage et des surfaces et l'ajustement des activités non agricoles. Trois profils d'organisation du travail sont identifiés : les "simplificateurs ", les " entrepreneurs " et les " autonomes ". Les variables discriminant ces profils sont le recours au salariat permanent et à l'entraide, ainsi que le degré de sophistication/ simplification de la conduite. Les profils, très peu liés aux terrains et aux caractéristiques structurelles et productives des exploitations, mettent en exergue des cohérences qui replacent la technique dans le système d'activité de travail des exploitants.
\end{abstract}

Mots clés : comparaison d'exploitation agricole ; exploitation agricole familiale ; organisation du travail ; zootechnie.

Thèmes : économie et développement rural ; mécanisation et aménagement rural ; systèmes agraires.

\section{Abstract \\ Work organisation in family livestock farms. Lessons from a comparative analysis of cases from France, South America, and Vietnam}

Interest in the future of family livestock farms fully justifies the study of work organisation on such farms, as a particular contextualisation of interactions between the composition of the farming family, the combination and dimensioning of economic activities (agricultural and non-agricultural) and the technical operation of the farm. Our study is based on a comparative analysis of 19 farms spread over four regions of the world (Pampas of Argentina and Uruguay; Brazilian Amazonia; French Segala; the mountains of North Vietnam), and chosen along with our local research and development partners. It aims at describing the diversity of forms of work organisation and at giving more food for thought about the future of livestock farming. The framework of our work analysis combines variables relating to the structural and productive characteristics of the livestock farms and others describing the configuration of the work unit, the arrangement of buildings and equipment, herd management and surface areas, and the adjustment of non-

\footnotetext{
* Pour citer cet article : Cournut S, Servière G, Hostiou N, Chauvat S, Dedieu B. L'organisation du travail en exploitations familiales d'élevage. Enseignements d'une analyse comparée conduite en France, en Amérique latine et au Vietnam. Cah Agric 2010 ; 19 : 1-10. DOI : 10.1684/agr.2010.0420.
} 
agricultural activities. Three work organization profiles are identified. The discriminating variables of the profiles are the recourse to permanent wage-earning, mutual aid, and the degree of sophistication/simplification of the management. The profiles, which are not closely related to the terrains or the structural and productive characteristics of the farms, underline points of coherence which replace technology in the farmers' work activity systems.

Key words: family farms; farm comparisons; work organization; zootechny.

Subjects: economy and rural development; farming systems; mechanization and rural planning.

e devenir des exploitations agricoles familiales est au cour des questions de développement durable des territoires ruraux (Gibon et Ickowicz, 2010). Cette catégorie " exploitation familiale " est cependant complexe, et couvre des réalités sociales, structurelles et techniques très diverses de par le monde, mais également au sein des pays. Le caractère largement familial du travail, c'est-à-dire l'existence d'un type particulier de relation non contractuelle, pas ou peu monétarisée, entre plusieurs des travailleurs formant la main-d'œuvre est une base reconnue de qualification de l'exploitation familiale (Cochet, 2008). D'autres éléments entrent également en ligne de compte, comme la propriété du capital ou encore l'importance d'une gestion patrimoniale (Petit, 2006; Chia et al., 2006). Engager une réflexion sur le devenir des exploitations familiales en lien avec les questions de développement des territoires implique d'analyser les interactions entre la composition de la famille, l'apport d'autres catégories locales de main-d'œuvre, la combinaison et le dimensionnement des activités économiques (agricoles et non agricoles) et le fonctionnement technique des activités agricoles (Laurent et al., 1998 ; Hoffman et al., 2007 ; Manoli et al., 2010). L'organisation du travail est une contextualisation particulière de ces interactions (Dedieu et al., 2006). Elle a pourtant peu fait l'objet d'études spécifiques visant à explorer ses caractéristiques et sa variabilité.

Nous développons dans cet article une étude des formes d'organisation du travail dans des exploitations familiales d'élevage d'herbivores. Cette étude s'appuie sur la mise en comparaison de 19 exploitations de différentes régions du monde dans les pays du Nord (Massif Central) et dans les pays du Sud (Amazonie brésilienne, Pampa d'Uruguay et d'Argentine, montagnes du Nord du
Vietnam). L'objectif de cette analyse comparative est de dégager des traits génériques de ce qui fonde la diversité des modes d'organisation (Lallement et Spurk, 2003). Les réalités socioproductives (c'est-à-dire le contexte social et l'organisation des filières) sont en effet a priori très variées, les systèmes économiques très contrastés: libéral en Amérique du Sud, dirigiste communiste au Vietnam et régulé en Europe. Nous considérons, en poursuivant le débat ouvert par Pichot (2006), que la réalité commune de l'exploitation familiale autorise un regard transversal, dans lequel les différences régionales ne seraient qu'un facteur de variation parmi d'autres de la diversité des logiques d'organisation et d'adaptation (Dedieu, 2009).

\section{Méthode}

\section{Enquêtes}

Cinq exploitations familiales d'élevage bovin (lait ou viande) ont été enquêtées dans les quatre terrains de recherche selon un protocole coconstruit avec les équipes étrangères partenaires (tableau 1). Sur le terrain Pampa, les enquêtes ont été réalisées dans les communes de Pigue (Argentine) et Salto (Uruguay), les conditions de production de ces deux régions et les formes d'élevage étant proches. En Amazonie brésilienne, les enquêtes ont été réalisées auprès d'éleveurs de bovin allaitant ou lait-viande sur le municipe d'Uruará localisé sur le front pionnier de la Transamazonienne. Au Vietnam, les éleveurs laitiers sont situés à Moc Chau en zone de montagne au nord du pays. Les éleveurs laitiers français ont été enquêtés dans le Ségala, petite région d'élevage intensif.
L'enquête, réalisée en une demi-journée avec les éleveurs, visait à : i) décrire la combinaison d'activités et les composantes structurelles de l'exploitation ainsi que le fonctionnement du système de production ; ii) décrire et comprendre le rôle de chaque travailleur au sein du collectif de travail via le type de tâches réalisées. Les temps de travaux n'ont pas été quantifiés car cela aurait nécessité une autre méthode d'enquête comme le Bilan travail plus coûteuse en temps (Diegez et al., 2010).

\section{Cadre d'analyse de l'organisation du travail}

Nous retenons le cadre d'analyse développé par la zootechnie des systèmes d'élevage (Dedieu et Servière, 2001 ; Madelrieux et Dedieu, 2008) pour lequel l'organisation du travail est considérée comme un système d'activités complexe, articulant quatre composantes : la maind'œuvre, les conduites d'élevage, les équipements et bâtiments et les autres activités (agricoles ou non). La maind'œuvre du noyau organisateur (l'exploitant seul, le couple, les associés) peut soit déléguer une partie des tâches soit les partager avec des bénévoles familiaux, des voisins dans le cadre de l'entraide, des salariés, ou des prestataires de services, par exemple, pour faire face à des pointes de travail (Harff et Lamarche, 1998). Les bâtiments et équipements influent sur les conditions de réalisation des activités, la sécurité, la vitesse et la précision d'exécution des tâches (Nielsen, 1999). L'ajustement du rythme ou du volume des activités extérieures peut également, dans un contexte de pluriactivité, infléchir l'organisation du travail agricole (Fiorelli et al., 2007 ; Hostiou et al., 2010). Les choix concernant la conduite technique participent aussi de l'organisation du travail: une conduite définit un 


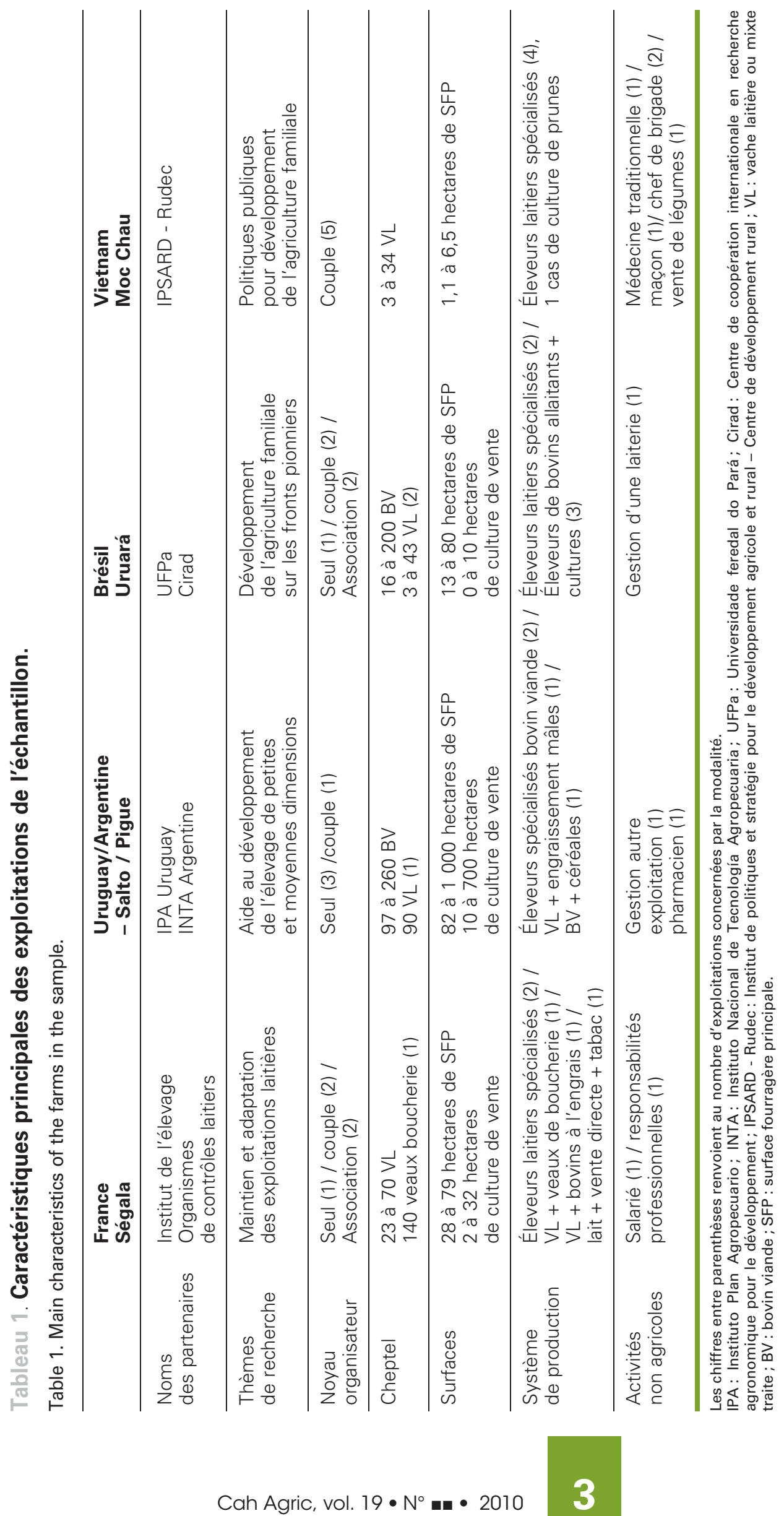


calendrier des tâches qui ont des caractéristiques temporelles (rythme et différabilité) et spatiales (distance, problèmes d'accès). Si, au sein d'un système agraire donné, les pratiques techniques et la durée du travail présentent une variabilité repérable, certaines pratiques dites "simplifiées" permettent d'alléger la durée de tâches à rythme quotidien (travail d'astreinte) ou de supprimer, voire regrouper, certaines tâches à rythme non quotidien (travaux de saison) (Madelrieux et Dedieu, 2008). Il en est ainsi par exemple des pratiques de monotraite quotidienne en élevage bovin laitier (Pomiès et al., 2008). Plusieurs études ont ainsi expliqué la cohérence de systèmes techniques, apparemment en décalage avec les recommandations d'optimisation économique, en analysant la contribution de pratiques simplifiées à la résolution de problèmes de travail (Cournut et Dedieu, 2005 ; Gleeson et al., 2008 ; Hostiou et Dedieu, 2009).

Plusieurs méthodes ont été développées pour rendre compte de l'agencement des composantes de l'organisation du travail à l'échelle d'une campagne agricole (Madelrieux et Dedieu, 2008). Nous faisons référence dans cet article aux approches de Cournut et Hostiou (2010), proposant de qualifier l'organisation du travail comme une combinaison de descripteurs simples de ces composantes.

\section{Variables}

Les variables définies et validées avec nos partenaires décrivent les caractéristiques structurelles et productives des exploita- tions et qualifient l'organisation du travail. Les structures sont décrites par la taille et la composition du collectif de travail familial qui gère l'exploitation (le noyau organisateur, NO), la combinaison et le dimensionnement des activités agricoles (cheptel, surface fourragère, cultures, type de production) et non agricoles de ce collectif. L'organisation du travail est qualifiée en référence aux quatre composantes évoquées ci-dessus (tableau 2). Sont pris en compte le parc matériel et bâtiment, la conduite du troupeau et de la surface fourragère, la main-d'œuvre hors noyau organisateur (bénévolat, entraide, salariat et entreprise), la répartition des tâches d'astreinte et de saison, l'ajustement - le cas échéant-de l'activité non agricole à certaines périodes de l'année.

\section{Tableau 2. Les variables relatives aux structures et aux composantes d'organisation du travail utilisées pour caractériser les élevages.}

Table 2. Variables about structure and forms of work organization used to characterize the farms.

\begin{tabular}{|c|c|c|}
\hline Libellé & Appréciation & Codification \\
\hline \multicolumn{3}{|c|}{ Caractéristiques structurelles et productives } \\
\hline Surface fourragère & Nombre d'hectares & 3 modalités $+=-$ en relatif intraterrain \\
\hline Cheptel & Nombre d'animaux & 3 modalités $+=-$ en relatif intraterrain \\
\hline Cultures & Nombre d'hectares & 3 modalités $+=-$ en relatif intraterrain \\
\hline Double activité & Temps annuel & 3 modalités $+=-$ en relatif intraterrain \\
\hline Type de production & Degré d'exigence en temps & 3 modalités $+=-$ en relatif intraterrain \\
\hline Noyau organisateur & Taille et composition & 3 modalités: seul, couple et associations \\
\hline \multicolumn{3}{|c|}{ Mobilisation des différentes composantes de I'organisation } \\
\hline Bénévolat & Sollicite le bénévolat familial & 2 modalités: oui/non \\
\hline Entreprise & Recours à l'entreprise & 2 modalités: oui/non \\
\hline Entraide & Recours à l'entraide & 2 modalités: oui/non \\
\hline Salariat temporaire & Embauche de journaliers/tâcherons & 2 modalités: oui/non \\
\hline Salariat permanent & Embauche de salarié permanent & 2 modalités: oui/non \\
\hline $\begin{array}{l}\text { Simplification } \\
\text { de la conduite animale }\end{array}$ & Degré de simplification & 3 modalités $+=-$ en relatif intraterrain \\
\hline $\begin{array}{l}\text { Simplification } \\
\text { de la conduite des surfaces }\end{array}$ & Degré de simplification & 3 modalités $+=-$ en relatif intraterrain \\
\hline Mécanisation & $\begin{array}{l}\text { Importance de la mécanisation } \\
\text { en propriété propre }\end{array}$ & 3 modalités $+=-$ en relatif intraterrain \\
\hline Bâtiments & Importance et fonctionnalité des bâtiments & 3 modalités $+=-$ en relatif intraterrain \\
\hline Répartition TA & $\begin{array}{l}\text { Degré d'autonomie de réalisation } \\
\text { du TA par le NO }\end{array}$ & 3 modalités : $\boldsymbol{A}$ autonomie, $\boldsymbol{P}$ partage, $\boldsymbol{D}$ délégation \\
\hline Répartition TST & $\begin{array}{l}\text { Degré d'autonomie de réalisation } \\
\text { du TST par le NO }\end{array}$ & 3 modalités : $\boldsymbol{A}$ autonomie, $\boldsymbol{P}$ partage, $\boldsymbol{D}$ délégation \\
\hline Répartition TSF & $\begin{array}{l}\text { Degré d'autonomie de réalisation } \\
\text { du TSSF par le NO }\end{array}$ & 3 modalités : $\boldsymbol{A}$ autonomie, $\boldsymbol{P}$ partage, $\boldsymbol{D}$ délégation \\
\hline $\begin{array}{l}\text { Ajustement entre activités } \\
\text { non agricoles et d'élevage }\end{array}$ & Quelle activité prime & $\begin{array}{l}3 \text { modalités : } \boldsymbol{A g} \text { agricole prime, } \\
\boldsymbol{C} \text { les deux coexistent, } \boldsymbol{N a g} \text { non agricole prime }\end{array}$ \\
\hline
\end{tabular}

$+=-$ : classement des modalités selon trois niveaux en se fondant sur la diversité observée au sein des 5 exploitations de chaque terrain ;

NO : noyau organisateur; TA : travail d'astreinte; TST : travail de saison sur le troupeau; TSSF : travail de saison sur les surfaces fourragères. 
Les variables ont ensuite été codifiées pour permettre l'analyse comparative en évitant un effet "pays " trop important : les valeurs brutes des critères de structure sont très différentes selon les pays (tableau 1). De la même façon, le niveau d'équipement ou la qualification d'une conduite dite simplifiée du point de vue du travail ne s'appuient pas sur les mêmes points de repères selon les pays. À Moc Chau, par exemple, posséder une moto pour le transport de l'affouragement correspond à un haut niveau de mécanisation dans une région où l'utilisation de buffles de trait domine. De même, dans la Pampa, un système fourrager constitué de prairies naturelles divisées en quelques parcelles exploitées en pâturage continu constitue une conduite simplifiée vis-à-vis de systèmes largement fondés sur des fourrages cultivés, prairies temporaires, sorgho fourrager (pour l'été) et avoine en vert (pour l'hiver). Ainsi, nous avons codé en relatif intraterrain les variables présentées au tableau 2 selon trois classes "-, =, + ", en raisonnant sur la diversité constatée. Ainsi, par exemple, sont réunies dans la même catégorie (+) des exploitations de 6,5 hectares au Vietnam et de 1700 hectares dans la Pampa, car toutes les deux sont "grandes " relativement à la variabilité de leur échantillon local. La configuration est, dans tous les terrains, fondée sur la famille nucléaire. Pour rendre compte de la configuration du collectif de travail et de la répartition du travail entre les différents membres, nous avons retenu :

- une variable binaire codée en deux modalités (présence/absence) par type de main-d'œuvre hors noyau organisateur (bénévolat, entraide, entreprise, salarié permanent et salarié temporaire qu'il soit tâcheron ou journalier);

- une variable par grand type de travaux (travail d'astreinte [TA], travail de saison troupeau [TST] et travail de saison surfaces fourragères [TSSF]), codée selon trois modalités : réalisation par le $\mathrm{NO}$ en autonomie totale, en partage avec une personne hors NO (salarié, bénévole), en partage et délégation avec une personne hors NO.

\section{Analyse des données}

Dans un premier temps, nous avons décrit les modalités d'organisation relatives à chaque composante (maind'œuvre, conduite, mécanisation et combinaison d'activités) et mis en évidence les différences et similarités entre terrains.
Dans un second temps, nous avons analysé la combinaison de ces composantes en utilisant une méthode d'analyse graphique qui amplifie la cognition visuelle (Bertin, 1977). Nous avons créé une matrice avec les exploitations étudiées en colonne, et les variables d'organisation du travail et leurs modalités en ligne (tableau 2). Nous avons rapproché les exploitations présentant des modalités similaires, repérées visuellement par permutation successive des lignes et des colonnes. Nous avons ainsi identifié trois profils d'organisation du travail (figure 1).

Afin de pouvoir traiter d'un lien éventuel entre caractéristiques structurelles et productives des exploitations et leur forme d'organisation du travail, nous avons parallèlement réalisé une typologie (analyse en composantes principales [ACP] suivie d'une classification ascendante hiérarchique $[\mathrm{CAH}]$ ) des exploitations selon ces caractéristiques (tableau 2). Quatre groupes typologiques transversaux aux terrains ont été définis. Leurs qualificatifs réfèrent aux critères dominants : " les petits élevages ", les " doubles actifs ", les " grands élevages " et les "polycultures-élevage ".

\section{Résultats}

\section{Composantes de l'organisation du travail}

Nous présentons tout d'abord la diversité des situations en analysant les modalités observées de chaque composante de l'organisation du travail dans les différents terrains.

\section{Force de travail}

Le bénévolat est présent dans la plupart des exploitations. Dans celles du Ségala, il s'agit d'un parent retraité souvent présent en permanence sur la ferme (Cournut et al., 2008) et donc sollicité pour réaliser du travail d'astreinte concernant le troupeau laitier (traite, surveillance, etc.). Dans les exploitations des autres terrains, le bénévolat est surtout le fait d'enfants scolarisés ou travaillant à l'extérieur : il est alors régulier (le week-end) ou périodique (congés scolaires ou pointes de travail agricole). Ils peuvent contribuer au travail d'astreinte et au travail de saison sur la surface fourragère. Le recours à l'entreprise pour le travail du sol ou les récoltes est fréquent dans les exploita- tions du Ségala et de la Pampa, mais absent dans celles de Moc Chau et d'Amazonie.

L'entraide entre agriculteurs, pour réaliser les chantiers de récolte de stocks fourragers, notamment les ensilages, nécessite une densité minimum d'exploitations dans le voisinage. On la retrouve dans le Ségala et à Moc Chau, deux terrains à très forte densité agricole. L'entraide est associée à l'utilisation de machines en commun (via les coopératives d'utilisation du matériel agricole, CUMA) en France ou à un chantier manuel très exigeant en main-d'œuvre au Vietnam (plusieurs dizaines de personnes pour l'ensilage de maïs). Elle est mineure dans les exploitations de la Pampa car la grande taille des exploitations induit une faible densité d'exploitations qui rend difficile le travail en commun entre voisins. Elle est peu fréquente en Amazonie, sauf pour la surveillance des brûlis des prairies envahies par la végétation adventice ou des surfaces forestières défrichées.

Le salariat renvoie à des réalités très contrastées (Madelrieux et al., 2010). Dans notre échantillon, les rythmes d'intervention et modalités de l'emploi sont les principaux facteurs de variation. Dans le Ségala, des salariés de groupement d'employeurs, de service de remplacement, de CUMA avec chauffeur peuvent intervenir soit en cas de coup dur, de congés, soit de façon régulière dans les exploitations. Les salariés peuvent être des tâcherons ou des journaliers pour les travaux de saison en Amazonie (sarclage des prairies, défriche forestière) et au Vietnam (chantiers de récolte, labours). Le salariat permanent toute l'année, observé dans les exploitations vietnamiennes et de la Pampa ainsi que dans une ferme en Amazonie brésilienne, est absent dans les exploitations du Ségala. Ainsi, partout en plus du bénévolat, l'exploitation familiale a recours ponctuellement ou plus structurellement, à une force de travail qui n'est pas familiale, pour partager le travail ou s'en libérer en délégant l'exécution de tâches. Dans tous les terrains, certains agriculteurs recherchent une autonomie dans le travail, alors que d'autres misent fortement sur la délégation partielle.

\section{Conduite des troupeaux et des surfaces fourragères et réduction du travail d'astreinte et/ou de saison}

Tous les terrains ne présentent pas la même diversité de conduite des trou- 


\begin{tabular}{|c|c|c|c|c|c|c|c|c|c|c|c|c|c|c|c|c|c|c|c|}
\hline & \multicolumn{6}{|c|}{ Simplificateurs } & \multicolumn{7}{|c|}{ Entrepreneurs } & \multicolumn{6}{|c|}{ Autonomes } \\
\hline Groupe typo * & POLY & DA & PE & $\mathrm{PE}$ & $\mathrm{PE}$ & POLYF & POLY & GE & DA & DA & DA & PE P & POLY & $\overline{P O L Y}$ & GE & DA & PE & DA & GE \\
\hline Élevage ** & S4 & S5 & P2 & S2 & A1 & $\mathrm{A} 2$ & V2 & V1 & V5 & A5 & P4 & P5 & P1 & A3 & A4 & S1 & V3 & V4 & S3 \\
\hline Bénévolat & $\mathrm{O}$ & $\mathrm{O}$ & $\mathrm{N}$ & $\mathrm{N}$ & $\mathrm{O}$ & $\mathrm{N}$ & $\mathrm{N}$ & $\mathrm{O}$ & $\mathrm{O}$ & $\mathrm{N}$ & $\mathrm{N}$ & $\mathrm{O}$ & $\mathrm{O}$ & $N$ & $\mathrm{~N}$ & $\mathrm{O}$ & $\mathrm{N}$ & $\mathrm{N}$ & $\mathrm{N}$ \\
\hline Entreprise & $\mathrm{O}$ & $\mathrm{O}$ & 0 & $\mathrm{O}$ & $\mathrm{N}$ & $\mathrm{N}$ & $\mathrm{N}$ & $\mathrm{N}$ & $N$ & $\mathrm{~N}$ & 0 & $\mathrm{~N}$ & $\mathrm{O}$ & $N$ & $\mathrm{~N}$ & $\mathrm{~N}$ & $\mathrm{~N}$ & $\mathrm{~N}$ & $\mathrm{O}$ \\
\hline Entraide & $\mathrm{O}$ & 0 & 0 & $\mathrm{O}$ & 0 & $\mathrm{O}$ & $\mathrm{O}$ & 0 & 0 & $\mathrm{~N}$ & $\mathrm{~N}$ & $\mathrm{~N}$ & $N$ & $N$ & $\mathrm{~N}$ & $\mathrm{O}$ & $\mathrm{O}$ & 0 & 0 \\
\hline Salariat temporaire & $\mathrm{O}$ & $\mathrm{N}$ & 0 & $\mathrm{O}$ & $\mathrm{O}$ & $\mathrm{O}$ & $\mathrm{O}$ & 0 & 0 & 0 & 0 & $\mathrm{O}$ & $N$ & $\mathrm{O}$ & 0 & $\mathrm{~N}$ & $\mathrm{~N}$ & $\mathrm{O}$ & 0 \\
\hline Salariat permanent & $N$ & $\mathrm{~N}$ & $\mathrm{~N}$ & $\mathrm{~N}$ & $\mathrm{~N}$ & $\mathrm{~N}$ & $\mathrm{O}$ & $\mathrm{O}$ & $\mathrm{O}$ & $\mathrm{O}$ & 0 & $\mathrm{O}$ & O & $N$ & $\mathrm{~N}$ & $\mathrm{~N}$ & $\mathrm{~N}$ & $N$ & $\mathrm{~N}$ \\
\hline $\begin{array}{c}\text { Simplification } \\
\text { de la conduite } \\
\text { animale }\end{array}$ & + & + & + & + & $=$ & $=$ & $=$ & $=$ & $=$ & $=$ & - & - & - & $=$ & & $=$ & $=$ & $=$ & - \\
\hline $\begin{array}{l}\text { Simplification } \\
\text { de la conduite } \\
\text { des surfaces }\end{array}$ & $=$ & + & + & + & + & + & $=$ & $=$ & $=$ & - & - & - & - & $=$ & $=$ & - & $=$ & $=$ & - \\
\hline Mécanisation & + & $=$ & $=$ & $=$ & - & - & + & + & - & - & - & - & + & - & - & $=$ & $=$ & - & $=$ \\
\hline Bâtiments & + & + & - & $=$ & - & - & - & - & - & - & - & - & - & - & - & - & - & - & + \\
\hline Répartition TA & $A$ & A & $A$ & $A$ & A & $A$ & $P$ & $P$ & $A$ & $P$ & $\mathrm{D}$ & $\mathrm{P}$ & $\mathrm{D}$ & $A$ & A & $A$ & A & $A$ & $A$ \\
\hline Répartition TST & $\mathrm{D}$ & $\mathrm{D}$ & $\mathrm{P}$ & $\mathrm{D}$ & A & $P$ & $P$ & $P$ & $P$ & $\mathrm{D}$ & $\mathrm{D}$ & A & $\mathrm{D}$ & $P$ & $\mathrm{P}$ & $A$ & A & $P$ & $\mathrm{D}$ \\
\hline Répartition TSF & $A$ & $A$ & $\mathrm{P}$ & $A$ & A & $P$ & $A$ & $A$ & $A$ & $\mathrm{D}$ & $\mathrm{D}$ & $P$ & $\mathrm{D}$ & A & A & $A$ & A & $A$ & $A$ \\
\hline $\begin{array}{c}\text { Ajustement } \\
\text { entre activités } \\
\text { non agricoles } \\
\text { et d'élevage }\end{array}$ & C & C & $\mathrm{C}$ & C & C & C & C & C & $\mathrm{Ag}$ & $\mathrm{Ag}$ & $\mathrm{Ag}$ & C & C & C & C & Nag & C & Nag & C \\
\hline
\end{tabular}

Figure 1. Tableau de Bertin et les trois profils d'organisation du travail pour les exploitations étudiées.

Figure 1. Bertin's table and third work organisation profiles for the studied farms.

* Groupe typologique issu de l'analyse des données structurelles et productives des exploitations : POLY : les polyculture-élevage ; DA : doubles actifs ; $\mathrm{PE}$ : petits élevages; GE : grands élevages.

** numéro d'élevage : la première lettre indique le terrain d'appartenance. A : Amazonie, P : Pampa ; S : Ségala ; V : Vietnam.

Les autres variables sont codées selon les modalités définies dans le tableau 2.

peaux et des surfaces fourragères. L'échantillon de Moc Chau est de loin celui qui présente la plus grande homogénéité d'itinéraires techniques, du fait d'une intégration forte de la production par la Compagnie laitière qui uniformise les pratiques d'élevage et de commercialisation (Hostiou et al., 2010). Les cinq troupeaux laitiers sont conduits en zéropâturage toute l'année, avec coupe quotidienne de fourrages cultivés et distribution d'ensilage de maïs en hiver. Dans les élevages amazoniens, la conduite du cheptel présente également une faible variabilité car les troupeaux 
sont conduits au pâturage toute l'année sans constitution de stock fourrager, et sans période de reproduction gérée par l'éleveur. Cependant, le mode d'entretien des prairies cultivées est hétérogène avec un impact sur les temps de travaux (Hostiou et Dedieu, 2009). Certains éleveurs entretiennent régulièrement leurs parcelles en éliminant les adventices alors que d'autres n'interviennent qu'au bout de plusieurs années en retournant les prairies dégradées.

La diversité des modes de conduite est beaucoup plus forte dans la Pampa où le système naisseur traditionnel d'élevage bovin allaitant (premier vêlage à 4 ans) fondé sur l'exploitation extensive de prairies naturelles uniquement pâturées coexiste avec des systèmes naisseurengraisseur plus intensifs, recherchant un premier vêlage à 2 ans, et engraissant des bœufs au grain et à l'ensilage de sorgho produit sur l'exploitation. Il en est de même dans les exploitations du Ségala, où sont distingués des systèmes traditionnels avec vêlages à l'automne et une alimentation reposant sur une forte proportion de maïs (15 à $20 \%$ de maïs), et d'autres systèmes groupant très fortement les vêlages de façon à fermer la salle de traite quelques semaines ou pratiquant la monotraite quotidienne en fin de lactation et fondés sur une valorisation maximale du pâturage. Là encore, les implications nettes des choix de conduites sur les temps de travaux et leur répartition dans le calendrier ont été largement documentées, notamment par l'Institut de l'élevage (Brocard et al., 2007).

Une mécanisation plus ou moins avancée et des bâtiments sommaires

Dans notre échantillon, les bâtiments sont très limités, à l'exception notable du Ségala, et ne discriminent donc pas les exploitations. La mécanisation est, relativement aux réalités locales, i) très variable dans les exploitations de la Pampa et du Vietnam, ii) un peu moins hétérogène dans le Ségala, et iii) sans objet à Uruará où le travail demeure manuel.

À Moc Chau, l'éleveur qui, en plus d'une moto, s'est équipé d'un pot trayeur et d'une coupeuse d'herbe motorisée portée à dos, dispose d'un haut niveau de mécanisation (et donc réduit son temps de travail) comparativement à l'éleveur pratiquant la traite manuelle, la coupe manuelle de l'herbe et le transport de l'affouragement à l'auge avec un buffle. Dans la Pampa, le niveau d'équipement est directement lié à l'importance des cultures, le suivi de l'élevage nécessite seulement un cheval ou un "pick-up" pour la surveillance et surtout un parc de contention en bon état que toutes les exploitations possèdent. Dans le Ségala, l'équivalent "haut niveau de mécanisation" est l'exploitation qui dispose, en propre ou en CUMA, d'une puissance de traction très élevée, du semis à la récolte, pour les cultures de l'herbe et du maîs ainsi que d'une automatisation avancée du travail d'astreinte (distributeur automatique de concentrés ou de lait pour les veaux) et d'une salle de traite équipée d'un décrochage automatique.

Quels que soient les terrains, le niveau de mécanisation apparaît assez directement lié à la taille de l'exploitation. Notons qu'il s'agit plus d'une mécanisation justifiée par les cultures (fourragères mais aussi de vente) dans la Pampa, et par le travail d'astreinte quotidien à Moc Chau, l'échantillon du Ségala étant dans une situation intermédiaire.

\section{Combinaison d'activités}

La pluriactivité à l'échelle du ménage (Laurent et al., 1998) concerne la moitié des élevages de l'échantillon, et ce dans tous les terrains. Trois modalités d'ajustement entre activité d'élevage et activité non agricole sont répertoriées. Une première modalité est celle où l'activité agricole prime. L'activité non agricole est alors ajustée quand la charge en travail sur l'activité agricole est importante. Il s'agit, par exemple, d'une exploitation à Moc Chau où le mari suspend son activité de médecine traditionnelle en période de récoltes des stocks de fourrages pour travailler aux champs.

Les deux activités coexistent (modalité 2). L'activité non agricole peut être continue et constante dans l'année (activité de contremaître dans une exploitation en Argentine) ou située à une période de l'année sans concurrencer les travaux agricoles (l'exploitant devient maçon pendant l'hiver dans une exploitation vietnamienne).

La troisième modalité est celle où l'activité non agricole prime. Elle n'est jamais interrompue parce qu'elle rapporte souvent un revenu conséquent (comme la vente de fruits par la femme d'un éleveur vietnamien ou encore la gestion d'une laiterie par un éleveur amazonien). Une période de pointe du calendrier technique nécessite alors un recours à de la main-d'œuvre temporaire, le plus souvent salariée.

\section{Trois profils d'organisation du travail}

L'analyse de la combinaison des différentes composantes a permis d'identifier trois profils d'organisation du travail dont nous présentons les principales caractéristiques.

\section{Les simplificateurs: \\ simplification de la conduite \\ de l'élevage avec entraide \\ pour les récoltes (6 élevages)}

L'organisation du travail est caractérisée par le caractère simplifié de la conduite du troupeau et/ou des surfaces, et le recours à l'entraide pour la réalisation du travail de saison sur les surfaces. Le travail lié au troupeau est réalisé par le noyau organisateur de façon assez autonome (aide de membres bénévoles de la famille dans la moitié des cas). Le travail de saison sur les surfaces est partagé avec des voisins durant les chantiers de récolte ou d'ensilage. L'entreprise intervient dans quatre cas pour des travaux à façon. Ces élevages sont plutôt fortement mécanisés par rapport à la moyenne de l'échantillon.

\section{Les entrepreneurs : \\ conduite sophistiquée et salariat (7 exploitations)}

Tous les exploitants emploient un ou deux salariés permanents, et leurs conduites techniques sont sophistiquées, c'est-à-dire exigeantes en travail d'astreinte et/ou en travail de saison du fait du développement des cultures, de l'entretien des surfaces ou de l'abondance des stocks. Le noyau organisateur de petite taille (couple ou agriculteur seul) partage le travail d'astreinte concernant le troupeau avec le(s) salarié(s). Ils n'hésitent pas ( 5 cas sur 7) à recourir à des journaliers en cas de pointe de travail ou pour des tâches particulières (clôtures à PigueSalto, nettoyage des adventices à Uruará). Dans les exploitations pluriactives, l'organisation du travail pour l'élevage garantit la possibilité d'une bonne coexistence des activités.

Les autonomes: autonomie pour le troupeau et peu de recours à la main-d'œuvre extérieure pour les travaux sur les surfaces (6 exploitations)

Ce profil est caractérisé par un recours modeste à la main-d'œuvre hors noyau organisateur, limité à l'entraide ou au 
salariat pour le partage de chantiers saisonniers sur les surfaces. Le noyau organisateur composé d'au moins deux personnes (couples ou associés) réalise en autonomie totale le travail concernant le troupeau. Les conduites ne présentent pas de caractère spécifique eu égard à la variabilité observée dans l'échantillon. L'activité non agricole lorsqu'elle est présente (2 élevages) est interrompue lors des périodes de pointe de travail agricoles, tels que les chantiers d'ensilage.

\section{Trois profils d'organisation du travail identifiés sur tous les terrains}

Les trois profils d'organisation identifiés dans notre échantillon se différencient par le recours à l'entraide, au salariat permanent et par la simplification de la conduite du troupeau et/ou des surfaces. Nous n'avons pas pu mettre en évidence de lien simple entre les types de structure de production ("petits élevages", "doubles actifs ", " grands élevages " et "polycultures-élevage") et les profils d'organisation du travail. L'appartenance à un terrain ne détermine pas non plus le type d'organisation du travail, les trois profils se retrouvant sur les différents terrains (sauf pour Moc Chau où le type " simplificateur" est absent).

\section{Discussion}

\section{Retour sur la méthode}

Le premier point de discussion a trait à l'échantillon, moins représentatif qu'emblématique des thèmes de recherchedéveloppement de nos partenaires locaux (tableau 1) (Bommel et al., 2010). Les trois profils, forcément contingents des terrains et des 19 cas, sont moins intéressants que les variables qui les discriminent: le recours (ou non) à certaines catégories de main-d'œuvre et la simplification de la conduite, celle-ci amenant une réduction des temps de travaux.

Le deuxième point concerne la codification selon un mode d'écart à la situation moyenne du terrain qui permet de s'extraire de la diversité des données brutes. Cette option présente deux limites. L'expression de l'avis des experts s'appuie sur des ordonnancements et des découpages en classes dont nous ne pouvons vérifier la répétabilité. Nous avons réduit ce risque d'imprécision en assurant une mise en classe intraterrain par trois experts, dont deux connaissaient au moins un autre des terrains de l'étude. Selon les terrains, la variabilité intraclasse n'est pas équivalente ce qui, bien sûr, influe sur les bornes des trois classes. Par ailleurs, les gains de temps apportés par la mécanisation dans les situations d'élevage où le travail manuel domine (pot trayeur, moto) ne sont pas du même ordre que ceux permis par l'amélioration des installations de traite ou d'alimentation (Wagner et al., 2001 ; Rotz et al., 2003). Notre codification en trois classes minore certains écarts entre fermes.

Le dernier est relatif au cadre d'analyse choisi qui ne permet pas de prendre en compte l'influence de facteurs comme la configuration spatiale des unités de production ou encore la saisonnalité de marchés sur l'organisation du travail. Cette étude montre que ce cadre d'analyse robuste s'applique à des situations très contrastées et permet d'extraire de la diversité des cas des éléments caractéristiques de l'organisation du travail dans les exploitations familiales d'élevage. Une analyse comparative de ce type, c'est-à-dire fondée sur une méthodologie unique mise en œuvre par des équipes distantes les unes des autres, rend nécessaire cette robustesse.

\section{Déterminants des formes d'organisation du travail}

Si les formes d'organisation ne semblent pas liées aux terrains, le contexte social et l'organisation des filières de chaque pays influent toutefois sur les modalités possibles de chaque composante d'organisation du travail. Ainsi, la densité des exploitations agricoles influence le recours à l'entraide, ou encore la filière (qui peut imposer ses normes de production) joue sur la diversité des conduites d'élevage. La conjoncture économique et les conceptions du travail des éleveurs (Pichereau et Lemery, 2004 ; Commandeur, 2005 ; Dufour et Dedieu, 2010) interviennent aussi dans leurs choix d'organisation pour raisonner l'intérêt de la délégation par exemple (Errington et Gasson, 1996), ou l'intérêt du développement de l'activité agricole ou non agricole.

\section{L'analyse du travail permet d'enrichir le regard porté sur les systèmes techniques}

Les choix de conduite des troupeaux et des surfaces fourragères participent par leur variabilité à la construction de profils d'organisation du travail différenciés. Nous avons réduit ici le qualificatif de "simplifié " à une modalité d'un facteur (la conduite) jouant sur la réduction des temps de travaux d'astreinte. La variabilité de la durée du travail d'astreinte par unité de bétail est en effet très importante avec une échelle de variation de 1 à 4, voire plus, selon les études (Olaizola et al., 2008 ; Cournut et Chauvat, 2010) : les marges de progrès sont importantes.

Cette lecture "travail " des systèmes techniques montre que les choix de conduite s'inscrivent dans un système d'activités de travail qui a sa propre cohérence pour rendre l'exercice d'élevage vivable. Les profils d'organisation "simplificateurs " et "entrepreneurs " suggèrent différentes voies d'évolution de l'élevage familial fondées l'un sur la maîtrise de la simplification, l'autre sur la recherche de performances poussées. Cette lecture éclaire la non-adoption de paquets techniques sophistiqués dans certaines exploitations (Mak, 2001; Rueda et al., 2003 ; Pardo et al., 2010), et incite même à considérer la mâtrise technique de la simplification comme une des voies pour concilier performances techniques et enjeux d'organisation du travail (Ferris et al., 2006). Les formules ambitieuses quant aux niveaux des performances recherchées laissent présager une voie possible d'évolution de l'élevage familial, vers les formes plus entrepreneuriales avec le recours de plus en plus important au salariat permanent et à la mécanisation, comme on le constate aujourd'hui, par exemple, en production porcine française et avicole (Salaün, 2008 ; Gallot, 2008) ou dans d'autres productions dans des pays où l'élevage s'industrialise (Wagner et al., 2001; Hadley et al., 2002). En tout cas, l'évolution de l'élevage familial nécessite la mise en place de recherches zootechniques appropriées et complémentaires de celles portant sur des modèles du type "mega farms" du Nord de l'Europe où la conduite n'obéit qu'à une rationalité technicoéconomique, alors que dans un contexte familial, nous montrons qu'il existe des cas où l'ajustement des conduites vers 
des formules moins exigeantes en travail permet de rendre compatible ce qui est à faire et les personnes - et les équipements - pour le faire.

\section{S'organiser pour faire face aux limitations d'une force de travail familiale}

Petit (2006) considère les exploitations familiales comme des systèmes efficients vis-à-vis de ressources rares, même si leurs performances sont, à première vue, en deçà de ce à quoi les modèles techniques performants permettent d'accéder. Notre étude suggère qu'elles ont une réelle capacité d'organisation pour faire face aux limitations de la force de travail familiale. Cette capacité de combinaison entre composantes de l'organisation du travail suggère des cohérences peu examinées dans les approches globales d'exploitations, telles que l'association entre la simplification de la conduite et le partage (ou la délégation) de travaux de saison, ou encore celle de l'autonomie en main-d'œuvre et la subordination de l'activité non agricole.

\section{Devenir des exploitations agricoles familiales et développement des territoires}

Notre étude n'a pas de prétention à une lecture diachronique de l'exploitation familiale. Tout au plus souligne-t-elle l'imbrication entre des réflexions sur les conduites techniques et leur évolution, et des réflexions sur les formes d'exercices de l'activité et sur les possibilités de développement d'activités non agricoles. Elle souligne également l'importance de la prise en compte de l'articulation, au niveau local, entre le salariat agricole et l'exploitation familiale, au-delà du paradoxe apparent entre les deux termes. Les salariés avec leur diversité de rythmes d'intervention, de statut et de compétences recherchées (Neiman, 2001 ; Madelrieux et al., 2010; Dupré, 2010) font bien partie du paysage de l'exploitation familiale, y compris dans les élevages plus ou moins nomades du Sénégal (Wane et al., 2010). Comment raisonner alors les implications d'une mutation forte des façons de faire de l'élevage, comme celle devant accompagner l'intensification écologique (Griffon, 2006), rendue nécessaire par les enjeux sur l'environnement ou sur la sécurité alimentaire? Nos observations suggèrent :

- d'une part, qu'un processus de redéfinition des schémas techniques ne fait pas sens tout seul mais doit être relié aux autres mutations locales, qui affectent les formes d'exercice de l'activité agricole et qui sont susceptibles d'interférer sur les systèmes d'activités des familles ;

- d'autre part, que ces schémas techniques devraient pouvoir être dotés d'une certaine plasticité (Beguin, 2007) pour être adoptés tant chez les " entrepreneurs " et les "autonomes" (qui devront alors réfléchir à l'évolution des compétences y compris de leur main-d'œuvre d'appoint salariée), que chez les "simplificateurs " qui chercheront des déclinaisons techniques moins exigeantes en travail.

\section{Conclusion}

Notre étude de la diversité de l'organisation du travail en exploitations familiales d'élevage montre qu'au-delà des différences de contextes sociaux et d'organisation des filières, les registres d'organisation sont les mêmes. Il existe bien une réalité commune de l'exploitation familiale d'élevage qui rend pertinente notre prise en compte du " travail " dans les systèmes d'élevage. Nous avons ainsi montré que les choix techniques avant d'être évalués en termes de performances productives ou environnementales, doivent être interprétés comme une contribution à un système de travail reliant l'activité d'élevage à la main-d'œuvre, aux autres activités et à la mécanisation. L'exploitation familiale ne saurait être caractérisée par la nature exclusivement familiale de la force de travail (même si celle-ci est bien un de ses fondamentaux). Une description un peu précise de la main-d'œuvre sollicitée souligne la participation d'autres personnes (pairs agriculteurs, salariés temporaires). Nous avons montré que l'organisation du travail était marquée par les formes de l'interaction entre l'activité agricole et d'éventuelles activités non agricoles, le devenir du salariat, l'entraide entre agriculteurs, c'est-à-dire tout un ensemble d'éléments qui caractérisent les liens entre l'exploitation et son territoire et qui doivent être analysés dans une perspective dynamique. Nos résultats s'ils ne permettent pas de conclure sur le lien entre organisation du travail des exploitations familiales d'élevage et performances productives et environnementales, donnent toutefois des éléments pour réfléchir l'évolution de ces exploitations vers plus de durabilité.

\section{Références}

Beguin P. Innovation et cadre socio-cognitif des interactions concepteurs-opérateurs : une approche développementale. Trav Humain 2007; 70 : 369-90.

Bertin J. La graphique et le traitement graphique de I'information. Paris: Flammarion, 1977.

Bommel P, Bah A, Etienne M, Leclerc G, Monteil C, Touré I. Vers un métamodèle pour analyser les systèmes d'élevage extensifs et leurs interactions avec les territoires. Cah Agric 2010; 19 : 143-51. Doi : 10.1684/agr.2010.0373

Brocard V, Portier B, Le Meur D, Porhiel JY, Lopez C. Traire les vaches une fois par jour ou grouper les vêlages: deux techniques pour réduire le travail d'astreinte en élevage. Renc Rech Rum 2007 ; 14 : 393-6.

Chia E, Dugué P, Sakho-Jimbira S. Les exploitations agricoles familiales sont-elles des institutions? Cah Agric 2006; 15: 498-505. Doi : 10.1684/agr.2006.0027

Commandeur M. Styles of pig farming and family labour in the Netherlands. J Comp Fam Stud $2005 ; 36$ : 391-8.

Cournut S, Chauvat S. Work organisation in livestock farms and farm liveability: Research findings from France. Neuvième IFSA Symposium, "Building sustainable rural future: The added value of systems approaches in times of change and uncertainty ", Vienne, 4-6 juillet 2010. http:// ifsa.boku.ac.at/cms/fileadmin/Proceeding2010/ 2010_WS2.3_Cournut.pdf

Cournut S, Dedieu B. Simplification des conduites d'élevage en bovins laitiers. Cah Agric 2005 ; $14: 541-7$.

Cournut S, Hostiou N. Adaptations des systèmes bovin laitier pour réduire la contrainte travail: une étude en Ségala (France). Cah Agric 2010; 19 : epub. Doi : 10.1684/agr.2010.0418

Cournut S, Hostiou N, Pailleux JY, Léger L. Quelles adaptations des systèmes laitiers pour réduire la contrainte "travail "? Renc Rech Rum 2008 ; $15: 163-6$.

Dedieu B. Qualification of the adaptive capacities of livestock farming systems. Rev Bras Zootecn $2009 ; 38: 397-404$.

Dedieu B, Servière G. Organisation du travail et fonctionnement des systèmes d'élevage. Renc Rech Rum 2001 ; 8 : 245-50.

Dedieu B, Servière G, Madelrieux S, Dobremez L, Cournut S. Comment appréhender conjointement les changements techniques et les changements du travail en élevage? Cah Agric 2006; 15 : 506-13. Doi : 10.1684/agr.2006.0028

Diegez F, Moralez H, Cournut S. La méthode Bilan travail pour l'approche du fonctionnement des élevages extensifs uruguayens. Cah Agric 2010 ; 19 : epub. Doi : 10.1684/agr.2010.0419

Dufour A, Dedieu B. Rapports au temps de travail et modes d'organisation en élevage laitier. Cah Agric 2010 ; 19 : epub. Doi : 10.1684/agr.2010.0422

Dupré L. Spécificités du salariat permanent en élevage laitier de montagne: une première approche dans les Alpes du Nord. Cah Agric 2010 ; 19 : epub. Doi : 10.1684/agr.2010.0423 
Errington A, Gasson R. The increasing flexibility of the farm and horticultural workforce in England and Wales. J Rural Stud 1996; 12: 127-41.

Ferris CP, Frost JP, Binnie RC, Patterson DC Dairy cows performance and labour inputs associated with two silage feeding systems. Grass Forage Sci 2006 ; 61 : 304-14.

Fiorelli C, Dedieu B, Pailleux JY. Explaining diversity of livestock farming management strategies of multiplejob holders: importance of level of production objectives and role of farming in the household. Animal 2007; 1 1209-18.

Gallot S. Le travail en aviculture - synthèse bibliographique. Paris: Institut technique de l'aviculture, 2008.

Gibon A, Ickowicz A. Transformations des systèmes d'élevage extensifs dans les territoires ruraux. Cah Agric 2010; 19 : 77-172.

Gleeson D, O'Brien B, O'Donovan K. The labour input associated with calf care on Irish dairy farms. Livest Sci $2008 ; 116: 82-9$.

Griffon M. Nourrir la planète. Pour une révolution doublement verte. Paris: Odile Jacob 2006.

Hadley GL, Harsh SB, Wolf CA. Managerial and financial implications of major dairy farm expansions in Michigan and Wisconsin. J Dairy Sci 2002 ; 85 : 2053-64.

Harff $Y$, Lamarche $H$. Le travail en agriculture: nouvelles demandes, nouveaux enjeux. Economie rurale $1998 ; 244$ : 3-11.

Hoffman MT, Allsopp N, Rohde RF. Sustainable land use in Namaqualand, South Africa: key issues in an interdisiplinary debate. J Arid Environ 2007; 70 : 561-9.

Hostiou N, Dedieu B. Diversity of forage system work and adoption of intensive techniques in dairy cattle farms of Amazonia. Agron Sustain Dev 2009 ; 29 : 535-44.
Hostiou N, Pham Duy K, Madelrieux S, Vu Trong $B$, Dedieu B. Relations entre organisation du travail et taille des exploitations laitières : une étude à Moc Chau (Vietnam). Cah Agric 2010； 19: epub. Doi : 10.1684/agr.2010.0404

Lallement M, Spurk J. Stratégies de la comparaison internationale. Paris : CNRS éditions, 2003.

Laurent C, Cartier S, Fabre C, Mundler P, Ponchelet $S$, Remy J. L'activité agricole des ménages ruraux et la cohésion économique et sociale. Economie Rurale 1998 ; 244 : 12-21.

Madelrieux S, Dedieu B. Qualification and assessment of work organisation in livestock farms. Animal 2008; 2 : 435-46.

Madelrieux S, Dupré L, Hostiou N, Barbosa T, Burlamaqui $A B$, Alves $A M$, Tourrand JF. Liens entre salariat et activité agricole: itinéraires professionnels de salariés d'élevage. Cah Agric 2010 ; 19 : epub. Doi : 10.1684/agr.2010.0426

Mak S. Continued innovation in a Cambodian rice-based farming system: farmer testing and recombination of new elements. Agr Syst 2001; 69 : $137-49$

Manoli C, Dedieu B, Ickowicz A. Livestock farming systems and local development: a review of the multiple dimensions of "Territory" livestock science. Neuvième IFSA Symposium, "Building sustainable rural future: The added value of systems approaches in times of change and uncertainty ", Vienne, 4-6 Juillet 2010. http:// ifsa.boku.ac.at/cms/fileadmin/Proceeding2010/ 2010_WS5.4_Manoli.pdf

Neiman G. Trabajo de campo. Produccion, technologia y empleo en el medio rural. Buenos Aires : Ediciones CICCUS, 2001.

Nielsen $V$. The effect of collaboration between cattle farms on the labour requirement and machinery costs. J Agr Eng Res 1999; 72: 197-203.

Olaizola AM, Chertouh T, Manrique E. Adoption of a new feeding technology in Mediterranean sheep farming systems : implications and economic evaluation. Small Ruminant Res 2008; 79: $137-45$
Pardo G, Riravololona M, Munier-Jolain NM Using a farming system model to evaluate cropping system prototypes: Are labour constraints and economic performances hampering the adoption of Integrated Weed Management? Eur J Agron 2010 : doi:10.1016/j.eja.2010.02.003

Petit M. L'exploitation agricole familiale: "lecons" actuelles de débats anciens. Cah Agric 2006 ; 15 : 486-90. Doi : 10.1684/agr.2006.0036

Pichereau F, Lemery B. Regards croisés du bilan travail et de l'analyse sociologique sur l'organisation du travail. Allocation à de grands troupeaux bovin viande. In : INRA \& Institut de l'élevage, eds. "Améliorer les conditions de travail en élevage ". Rencontres nationales des acteurs de la recherche-développement, Poitiers, 18-19 mars 2004

Pichot JP. L'exploitation agricole: un concept à revisiter du nord aux suds. Cah Agric 2006; 15 : 483-5.

Pomiès D, Marnet PG, Cournut S, Barillet F Guinard-Flament J, Rémond B. Les conduites de traite simplifiées en élevage laitier: vers la levée de l'astreinte biquotidienne. INRA Prod Anim 2008; 21 : 59-70.

Rueda BL, Blake RW, Nicholson CF, et al. Production and economic potentials of cattle in pasturebased systems of the western Amazon region of Brazil. J Anim Sci 2003 ; 81 : 2923-37.

Rotz CA, Coiner CU, Soder KJ. Automatic milking systems, Farm size, and milk production. J Dairy Sci $2003 ; 86: 4167-77$.

Salaün Y. Le travail en production porcine: synthèse bibliographique à partir des études conduites par I'Institut du porc et ses partenaires. Paris : IFIP, 2008

Wagner A, Palmer RW, Bewley J, Jackson-Smith DB. Producer Satisfaction, Efficiency, and Investment Cost Factors of Different Milking Systems. J Dairy Sci 2001 ; 84 : 1890-8.

Wane A, Ancey V, Diao-Camara A, Ndiobène Kâ S, Touré I. L'économie pastorale face aux incertitudes - Le salariat au Sahel du Sénégal. Cah Agric 2010; 19: epub. Doi: 10.1684/ agr.2010.0427. 